\title{
Comparative study of screening techniques used in early detection of urinary bladder cancer
}

\begin{abstract}
Background: Early detection of urinary bladder cancer can help improve patients' outcomes. Detection and follow-up for recurrence by cystoscopy and histopathology of obtained biopsies is costly and causes discomfort to patients. There is a need to find sensitive, specific screening techniques as alternatives to reduce the frequency or to improve the sensitivity for detection of early bladder cancer.
\end{abstract}

Objective: This study aimed to compare bladder washing cytology (BWC), bladder tumor antigen (BTA), flowcytometry (FCM), and photodynamic diagnosis (PDD) for early detection of urinary bladder cancer.

Methods: This prospective cohort study was conducted on 75 patients with hematuria/ cystitis or previous resection of bladder cancer. The four diagnostic techniques were performed in every patient. The results of the studied techniques were compared to the histopathological findings in these patients. Sensitivity, specificity, negative predictive value (NPV), and positive predictive value (PPV) were calculated.

Results: The BWC and PDD showed the highest accuracy (each was 77.3\%). The PPD technique had the highest sensitivity and NPV (91.3\% and $94.9 \%$, respectively), while the BWC had the highest specificity and PPV (92.3\% and 71.4\%, respectively). Sensitivity was higher in high grade tumors than the low grades in BWC, while sensitivity was higher in the low-grade tumors than the high grades in the other techniques.

Conclusions: The BWC and PDD had the highest accuracy for detection of bladder cancer. Their combined use is recommended to obtain high sensitivity and specificity.

Keywords: bladder wash cytology, bladder tumor antigen, flowcytometry, photodynamic diagnosis, urinary bladder cancer
Volume 7 Issue 6 - 2019

\author{
Sami A Abbas \\ Consultant Urologist; National Institute of Urology and \\ Nephrology; Cairo, Egypt
}

\begin{abstract}
Correspondence: Sami A Abbas, Consultant Urologist, National Institute of Urology and Nephrology, Cairo, Egypt, Emailsamiahmedabbas@yahoo.com
\end{abstract}

Received: November 22, 2019 | Published: December 23, 2019
Abbreviations: BWC, bladder washing cytology; BTA, bladder tumor antigen; FCM, flowcytometry; PDD, photodynamic diagnosis; NPV, negative predictive value; PPV, positive predictive value; CIS, carcinoma in situ; CT, computed tomography; TURBT, transurethral resection of superficial bladder tumor

\section{Introduction}

An important step in the management of the urinary bladder cancer is early detection before progression into invasive cancer if left untreated. ${ }^{1}$ Screening for bladder cancer in patients who present with hematuria or with irritative voiding symptoms is mandatory. ${ }^{2}$ Unfortunately, the sensitivity of voided urine cytology is $30 \%$ in low grade disease. Thus, the development of highly sensitive urinary tests for the detection of bladder cancer is required to avoid delayed management and improve patients' outcomes. ${ }^{3}$ Screening techniques are required to have acceptable sensitivity, specificity as well as positive and negative predictive values. A test with false positive result may lead to unnecessary expense and possible morbidity. Alternatively, a test with false negative result may deceive patients and their physicians with a false sense of security that may delay the diagnosis of cancer. ${ }^{4}$ Some promising screening techniques were developed for detection of bladder cancer. Urine cytology is considered for the detection of flat TCC and carcinoma in situ (CIS) lesions that may go unrecognized by cystoscopy. However, cytological preparations are more sensitive for detecting the highergrade lesions. Urine cytology is of benefit before cystoscopy in highly suspicious cases without obvious lesions by pelvic ultrasound or computed tomography (CT). ${ }^{5}$ The bladder tumor antigen (BTA) test detects basement membrane degradation fragments, which occur mainly in invasive disease. ${ }^{6}$ Flowcytometry (FCM) detects DNA aberrations (polyploidy or aneuploidy) that may appear at an early stage of the tumor development and before recognizable changes in morphology. ${ }^{7}$ Photodynamic diagnosis (PDD) of neoplastic urothelial bladder is an approach in which hexaminolevulinic acid is instilled locally in the bladder as a photosensitizer that can be used to label bladder tumors. This technique is particularly useful to reveal areas of dysplasia, CIS and micro papillary tumor. ${ }^{8}$ The aim of the present study was to compare the results of some screening tests for urinary bladder cancer (including urine cytology, BTA, FCM, and PDD) with the histopathological examination results of suspicious areas in screened at risk patients. 


\section{Methods}

\section{Ethical considerations}

The study protocol was approved by the Research Ethics Committee of the National Institute of Urology and Nephrology, Cairo, Egypt. Informed written consent was obtained from each patient. Patients' data were kept anonymous.

\section{Study settings}

This study was conducted on 75 patients who attended urology clinic during the period from January 2017 to February 2019.

\section{Eligibility criteria}

The study included patients of either sex who were 18- yearsold or above and presented with one of the following: a) persistent cystitis (after exclusion of urinary TB and urinary tract infection); b) hematuria (with no manifested cause); and c) transurethral resection of superficial bladder tumor (TURBT) or precancerous lesions.

\section{Preliminary evaluation}

All patients were subjected to initial evaluation, including a full history taking, meticulous general and abdominal examination, and bimanual digital rectal or vaginal examination. Investigations included urine analysis, full blood picture, blood sugar level, liver enzymes, prothrombin time and concentration, serum creatinine, blood urea level, the excretory urography (IVU), abdominopelvic ultrasonography and CT.

\section{Bladder wash cytology (BWC)}

A bladder wash sample was obtained prior to cystoscopy by rinsing the urinary bladder at least twice with $50 \mathrm{cc}$ saline solution. Part of the bladder wash sample was used for routine cytological examination by a pathologist. Each sample was cytologically scored as negative, atypia, low-grade or high-grade cancer.

\section{Bladder tumor antigen (BTA) test}

A voided urine sample was collected for BTA stat test after urinary tract infection was excluded. The patient's urine was added to the BTA test stat and allowed to react with the control zone and patient zone. In the patient (p) zone, antigen-conjugate complexes are captured by a second antibody forming a visible line. In the absence of the antigen in the patient urine, no visible line is formed. The control zone contains an immobilized reagent, which will capture the conjugate independently in the presence or absence of the antigen, thereby allows producing a line. This procedural control assures the operator that the device is working properly.

\section{Flowcytometry (FCM)}

Before cystoscopy, the bladder was washed vigorously 6 or7 times through a Nelaton catheter using approximately $200 \mathrm{ml}$ saline. The samples were centrifuged, then suspended in phosphate buffered saline followed by a second centrifugation and fixation in $50 \%$ ethanol, staining with propidium iodide and analysis with FCM equipped with an argon laser light beam (wavelength $488 \mathrm{~mm}$ ). ${ }^{9,10}$ For histograms with less than 10,000 cells, at least one clearly identifiable peak had to be present to consider the analysis interpretable. Samples not meeting these criteria were rejected. ${ }^{11}$

\section{Photodynamic diagnosis with fluorescence detection}

Cystoscopy was performed after intravesical instillation of hexaminolevulinic acid in patients with suspected primary or recurrent bladder cancer. Two to three hours before planned cystoscopy a neutral solution was prepared to contain $1.5 \mathrm{gm}$ of hexaminolevulinic acid dissolved in $50 \mathrm{ml} 2.4 \%$ sodium bicarbonate. This freshly prepared solution was instilled via 12-Frenchcatheter after evacuation of the bladder, and the catheter was then removed. The patient was asked to change his position regularly to obtain sufficient mucosal contact of the entire bladder surface. ${ }^{12}$ The dosimetry light system was used for fluorescence diagnosis. It consists of a light source with a xenon arc lamp and a corresponding band pass filter with a wave length of 380 to $450 \mathrm{~mm}$. By switching the foot pedal, the filter was placed or removed and either blue or standard white light was produced. ${ }^{13}$ The urinary bladder was first inspected under white light before exposure to blue light and every fluorescence positive area was once more inspected with a white light and macroscopic appearance was noted. Cold biopsies were taken from every fluorescent area, every macroscopically suspicious non-fluorescent area, and at least two normally appearing non-fluorescent zones. Hypervascularizd erythematous, erosive or edematous areas of mucosa were considered non-specifically inflamed.

\section{Statistical analysis}

Data were collected and analyzed using Statistical Package for Social Sciences, version 22. Numerical variables with normal distribution were summarized as mean \pm standard deviation. Categorical variables were summarized as frequencies. Sensitivity, specificity, NPV, and PPV for each score were determined in relation to histopathological examination of biopsies.

\section{Results}

Seventy-five patients (56males and 19females) with manifestations of superficial urinary bladder cancer or previously treated with TURBT were included in this study. The mean age of patients was 41.2 years for males and 42.2 years for females. Histopathological examination revealed that the urinary bladder lesions were benign in 52 patients $(69.3 \%)$ and malignant in 23 patients $(30.7 \%)$. The malignant lesions were of low grade in 13 patients (17.3\%), high grade in six patients $(7 \%)$, and invasive in four patients $(5.3 \%)$ (Table 1). The results of the studied screening tests were compared to the findings of histopathological examination and accordingly the frequencies of true positive, false positive, true negative and false negative results of each test were recorded. A positive result of a test indicates a malignant lesion, while a negative result indicates a benign lesion. From these data, sensitivity, specificity, PPV, NPV, and overall accuracy of each test were calculated (Table 2). The techniques with the highest overall accuracy were BWC and PDD (each was 77.3\%). The PPD technique had the highest sensitivity and NPV $(91.3 \%$ and $94.9 \%$, respectively), while the BWC had the highest specificity and PPV (92.3\% and $71.4 \%$, respectively). The sensitivity of each screening technique was also studied in relation to the grading of the malignant lesion (Table 3). For BWC, the sensitivity was $30.8 \%, 50 \%$, and $75 \%$ in low, high grade, and invasive lesions, respectively. For BTA, the sensitivity was $84.6 \%, 66.7 \%$, and $100 \%$ in low, high grade, and invasive lesions, respectively. For FCM, the low number of true positive results interferes with the interpretation of the sensitivity in different grades of malignancy. For PDD, the sensitivity was $92.3 \%$, $100 \%$, and $75 \%$ in low, high grade, and invasive lesions, respectively. 
Table I Distribution of sex and age of the studied cases

\begin{tabular}{|c|c|c|c|}
\hline \multicolumn{4}{|c|}{ Patients' characteristics (total $n=75$ ) } \\
\hline \multicolumn{4}{|l|}{$\operatorname{Sex}^{\mathrm{a}}$} \\
\hline & Females & 19 & 25.3 \\
\hline & Males & 56 & 74.7 \\
\hline \multicolumn{4}{|l|}{ Age (years) ${ }^{b}$} \\
\hline & All patients & \multicolumn{2}{|c|}{$41.8(17-84)$} \\
\hline & Females & \multicolumn{2}{|c|}{$42.2(17-70)$} \\
\hline & Males & \multicolumn{2}{|c|}{$41.2(17-84)$} \\
\hline \multicolumn{4}{|c|}{ Histopathology findings ${ }^{\mathrm{a}}$} \\
\hline Benign & & 52 & 69.3 \\
\hline Malignant & & 23 & 30.7 \\
\hline \multirow[t]{2}{*}{ Low Grade } & Dysplasia & 10 & 13.3 \\
\hline & $\mathrm{TaGl}$ & 3 & 4 \\
\hline \multirow[t]{3}{*}{ High Grade } & TaGIII & 3 & 4 \\
\hline & TIGII & 2 & 2.7 \\
\hline & TIGIII & 1 & 1.3 \\
\hline \multirow[t]{2}{*}{ Invasive } & T2GII & 3 & 4 \\
\hline & T3GII & $\mathrm{I}$ & 1.3 \\
\hline
\end{tabular}

Table 2 The sensitivity, specificity, positive and negative predictive values of the studied screening techniques for detection of malignant urinary bladder lesions in the study patients

\begin{tabular}{|c|c|c|c|c|}
\hline & \multicolumn{3}{|c|}{$\begin{array}{l}\text { The studied screening } \\
\text { techniques }\end{array}$} & \multirow[b]{2}{*}{ PDD } \\
\hline & BWC & BTA & FCM & \\
\hline True positive results (n) & 10 & 19 & 4 & 21 \\
\hline False positive results ( $n$ ) & 4 & 32 & 5 & 15 \\
\hline False negative results ( $n$ ) & 13 & 4 & 19 & 2 \\
\hline True negative results ( $\mathrm{n}$ ) & 48 & 20 & 47 & 37 \\
\hline Sensitivity (\%) & 43.5 & 82.6 & 17.4 & 91.3 \\
\hline Specificity (\%) & 92.3 & 38.5 & 90.4 & 71.2 \\
\hline PPV (\%) & 71.4 & 37.3 & 44.4 & 58.3 \\
\hline NPV (\%) & 78.7 & 83.3 & 71.2 & 94.9 \\
\hline Overall accuracy (\%) & 77.3 & 52 & 68 & 77.3 \\
\hline
\end{tabular}

BWC, bladder wash cytology; BTA, bladder tumor antigen; FCM, flowcytometry; PDD, photodynamic diagnosis; n: number; PPV, positive predictive value; NPV, negative predictive value

a: number and percentage; b: mean (range)

Table 3 The sensitivity, specificity, positive and negative predictive values of the studied screening techniques for detection of malignant urinary bladder lesions in the study patients

\begin{tabular}{|c|c|c|c|c|c|c|c|c|c|c|}
\hline \multicolumn{2}{|c|}{ Histopathology } & \multirow[t]{2}{*}{$\begin{array}{l}\text { Total } \\
\text { number }\end{array}$} & \multicolumn{2}{|l|}{ BWC } & \multicolumn{2}{|l|}{ BTA } & \multicolumn{2}{|l|}{ FCM } & \multicolumn{2}{|l|}{ PDD } \\
\hline & & & $T P(n)$ & $\begin{array}{l}\text { Sensitivity } \\
(\%)\end{array}$ & $T P(n)$ & $\begin{array}{l}\text { Sensitivity } \\
\text { (\%) }\end{array}$ & $T P(n)$ & $\begin{array}{l}\text { Sensitivity } \\
(\%)\end{array}$ & $\mathbf{T P}(\mathbf{n})$ & $\begin{array}{l}\text { Sensitivity } \\
\text { (\%) }\end{array}$ \\
\hline \multirow[t]{3}{*}{ Low Grade } & Dysplasia & 10 & 2 & 20 & 9 & 90 & 0 & 0 & 9 & 90 \\
\hline & $\mathrm{TaGl}$ & 3 & 2 & 66.7 & 2 & 66.7 & I & 33.3 & 3 & 100 \\
\hline & Total & 13 & 4 & 30.8 & II & 84.6 & I & 7.7 & 12 & 92.3 \\
\hline \multirow[t]{4}{*}{ High Grade } & TaGIII & 3 & I & 33.3 & 3 & 100 & I & 33.3 & 3 & 100 \\
\hline & TIGII & 2 & 2 & 100 & 0 & 0 & I & 50 & 2 & 100 \\
\hline & TIGIII & I & 0 & 0 & I & 100 & 0 & 0 & I & 100 \\
\hline & Total & 6 & 3 & 50 & 4 & 66.7 & 2 & 33.3 & 6 & 100 \\
\hline \multirow[t]{3}{*}{ Invasive } & T2GII & 3 & 2 & 66.7 & 3 & 100 & I & 33.3 & 3 & 100 \\
\hline & T3GII & I & 1 & 100 & I & 100 & 0 & 0 & 0 & 0 \\
\hline & Total & 4 & 3 & 75 & 4 & 100 & I & 25 & 3 & 75 \\
\hline
\end{tabular}

BWC, bladder wash cytology; BTA, ladder tumor antigen; FCM, Flowcytometry; PDD, photodynamic diagnosis; TP, true positive 


\section{Discussion}

In the present study, we compared four of the most commonly used screening techniques to detect bladder cancer. The BWC is popularly used as it is superior to both voided urine sample (due to its hypercellularity) and catheterized sample (due to better preservation of cells). ${ }^{14}$ The sensitivity of BWC for detection of malignant lesions was $43.5 \%$ and the specificity was $92.3 \%$. The sensitivity of BWC was $30.8 \%$ in low grade tumors, $50 \%$ in high grade tumors, and $75 \%$ in invasive tumors. These results were in partial agreement with those of Kranzbühler et al. ${ }^{15}$ who found that the overall sensitivity and specificity were $16 \%$ and $99 \%$, respectively. The sensitivity was $13 \%$ for low grade, and $20 \%$ for high grade disease. In this study, the overall sensitivity of BTA test was $82.6 \%$, meanwhile the sensitivity of the test was $84.6 \%, 66.7 \%$, and $100 \%$ for low grade, high grade, and invasive tumors, respectively. Other studies reported an overall sensitivity ranging from $56 \%$ to $83 \%$ sensitivity. ${ }^{16-20}$ This great variability of the results might be caused by differences in sample size, the proportion of new cases versus recurrent tumor as well as grades and stages of the tumors. The specificity of BTA test in our study was $38.5 \%$, which is much lower than that reported previously $(72 \%-92 \%) .{ }^{16-20}$ This lower specificity could be attributed to the high frequency of false positive results in our study that may have been caused by the intravesical instillation of BCG in 32 cases before starting the study. In addition, the presence of benign schistosomal lesions or chronic bladder disease may cause false positive results. Similarly, Raitanen ${ }^{17}$ reported that the specificity of BTA test dropped in patients treated with BCG due to BCG-induced local inflammatory effect.

The relatively high false positive result rates may be explained by the fact that urine samples may be extremely hypocellular in some patients with bladder neoplasms. ${ }^{7}$ Moreover, false positives may occur due to hematuria as human complement factor H-related protein is present in blood. Urological conditions that may present with hematuria - and thus give rise to false positive results - include urolithiasis, recent instrumentation, inflammation, other genitourinary malignancies, and BCG therapy -induced cystitis. ${ }^{18,21}$ Therefore, the use of BTA tests cannot be recommended for use in clinical practice as its results showed low sensitivity and high rates of false-positive results. ${ }^{22}$ Our study revealed that the overall sensitivity of FCM analysis was $17.4 \%$. The sensitivity of FCM analysis for low grade bladder cancer was $7.7 \%$ and for high grade non-invasive bladder cancer was $33.3 \%$. However, its sensitivity for invasive bladder cancer decreased to $25 \%$ because we have 2 cases with persistent hematuria, which makes the test invalid. Meanwhile, the overall specificity of FCM analysis in our study was $90.4 \%$.

The sensitivity of FCM- as reported by previous studies - varied widely according to the tumor grade. The sensitivity of FCM ranged from $12 \%$ to $70 \%$ in low grade tumors and from $45 \%$ to $89 \%$ in high grade tumors. ${ }^{11}$ This is attributed to the cut off values for determining significant hyper-diploid fraction, which vary from $7 \%$ to $12 \%$ in these studies. This indicates the limited diagnostic value of DNA -FCM on bladder washing in case of low grade and low stage tumors. ${ }^{4}$ Similar disappointing results with diagnostic DNA FCM were reported. ${ }^{23}$ In patients with CIS, cytology and FCM are helpful in the diagnosis of these high grade lesions with a sensitivity of more than $90 \% .{ }^{24}$ The most frequent types of bladder cancer among our patients were low grade and of early stages. Consequently, FCM was of little value in screening for early detection of bladder cancer as it has usually diploid DNA content. In addition, intravesical chemotherapy and immunotherapy with BCG induce hypocellularity and inflammatory reaction that decrease the accuracy of $\mathrm{FCM}^{23}$ Fluorescence cystoscopy using PPD can detect neoplastic and pre-cancerous lesions among areas of healthy tissue, allowing more accurate sampling for histopathological examination and enabling removal of the tumor with an appropriate safety margin. ${ }^{25}$ In the current study, the overall sensitivity of PDD was $91.3 \%$. This is compatible with the results of previous studies that reported a range from $92 \%$ to $100 \%{ }^{26,27}$ The sensitivity of PDD for low grade tumors and dysplasia was 92.3, which is comparable to the results of Witjes et al. ${ }^{8}$ and Ray et al. ${ }^{28}$ ( $94 \%$ and $95 \%$, respectively). The sensitivity of PDD for high grade bladder tumors was $100 \%$, which is compatible with earlier studies. ${ }^{8,13}$

The sensitivity of PDD in our study was affected by a case of invasive bladder tumor that gave false negative result by PDD. This could be explained by the concealment of this lesion under a non-fluorescent fibrous scar following radiotherapy received before our examination. Sakel et al. ${ }^{12}$ demonstrated limitation of PDD after previous radiotherapy or at the previous TURBT site during secondary TURBT as they found its sensitivity to be $81 \%$ in their group of patients undergoing secondary TURBT. Unfortunately, a high incidence of false positive results has been found in the current study that decreased specificity in our series of patients to $71.2 \%$, which is comparable to the results of Kriegmair et al. ${ }^{29}(66 \%$ to $78 \%)$. The high percentage of false positives is mainly due to accumulation of the photosensitizer in tissues during inflammation. Aboumarzouk et al. ${ }^{30}$ reported a high percentage of false positives within less than 3 months following intravesical administration of BCG and after recent bladder resections. The data present in our study revealed that the best diagnostic tests were the BWC and PDD as both tests attained an overall accuracy of $77.3 \%$. The BWC had the highest specificity and PPV, while PDD had the highest sensitivity and NPV. These findings suggest that the combined use of both tests may be more helpful in early diagnosis of bladder cancer. In the current study, it was found that the overall sensitivity for BWC was $43.5 \%$ versus $82.6 \%$ for BTA test. This increased sensitivity of BTA test over BWC is more apparent in the lower grade tumors $(84.6 \% \mathrm{Vs} 30.8 \%$, respectively). On the other hand, this difference diminished slightly but was still apparent for high grade tumors ( $66.7 \%$ vs $50 \%$ for BTA test and BWC respectively). The sensitivities of FCM for low grade, high grade, and invasive bladder cancer were $7.7 \%, 33.3 \%$, and $25 \%$, respectively.

\section{Conclusion}

In conclusion, bladder wash cytology and BTA test are helpful in diagnosing high grade carcinoma, whereas low grade tumors often reveal false negative results. However, PDD-guided biopsies can be helpful for detecting low grade tumors. The combined use of BWC and PDD seems to provide the best results among the four studied techniques. Future studies with larger sample sizes are recommended to confirm the accuracy of each test with respect to the tumor grade and stage.

\section{Acknowledgments}

None.

\section{Conflicts of interest}

The author declares there is no conflict of interest. 


\section{References}

1. Stenzl A, Burger M, Fradet Y, et al. Hexaminolevulinate guided fluorescence cystoscopy reduces recurrence in patients with nonmuscle invasive bladder cancer. The Journal of urology. 2010;184(5):19071913

2. Millholland JM, Li S, Fernandez CA, et al. Detection of low frequency FGFR3 mutations in the urine of bladder cancer patients using nextgeneration deep sequencing. Research and reports in urology. 2012;4:33-40.

3. Mowatt G, Zhu S, Kilonzo M, et al. Systematic review of the clinical effectiveness and cost-effectiveness of photodynamic diagnosis and urine biomarkers (FISH, ImmunoCyt, NMP22) and cytology for the detection and follow-up of bladder cancer. Health technology assessment. 2010;14(4):1-331.

4. Goodison S, Rosser CJ, Urquidi V. Bladder cancer detection and monitoring: assessment of urine- and blood-based marker tests. Mol Diagn Ther. 2013;17(2):71-84.

5. Sullivan PS, Chan JB, Levin MR, et al. Urine cytology and adjunct markers for detection and surveillance of bladder cancer. Am J Transl Res. 2010;2(4):412-440.

6. Miyake M, Goodison S, Rizwani W, et al. Urinary BTA: indicator of bladder cancer or of hematuria. World J Urol. 2012;30(6):869-873.

7. Sunwoo HH, Suresh MR. Cancer markers. In: Wild D, editor. The immunoassay Hand book. $4^{\text {th }}$ ed. Great Britain: Elsevier Ltd; 2013. p. 833-856.

8. Witjes JA, Redorta JP, Jacqmin D, et al. Hexaminolevulinate-guided fluorescence cystoscopy in the diagnosis and follow-up of patients with non-muscle-invasive bladder cancer: review of the evidence and recommendations. Eur Urol. 2010;57(4):607-614.

9. Comploj E, Mian C, Ambrosini-Spaltro A, et al. uCyt+/ImmunoCyt and cytology in the detection of urothelial carcinoma. Cancer Cytopathology. 2013;121(7):392-397.

10. Fradet Y, Grossman HB, Gomella L, et al. A Comparison of Hexaminolevulinate Fluorescence Cystoscopy and White Light Cystoscopy for the Detection of Carcinoma in Situ in Patients with Bladder Cancer: A Phase III, Multicenter Study. Journal of Urology. 2007;178(1):68-73.

11. Wadhwa N, Jatawa SK, Tiwari A. Republished: non-invasive urine based tests for the detection of bladder cancer. Postgrad Med J. 2013;89(1052):352-327.

12. Sakel AA, Mazdar A, N'Guibou F. Rôle de la cystoscopie de fluorescence en lumière bleue induite par Hexvix dans la prise en charge des tumeurs urothéliales vésicales. Journal de l'Association des urologues du Canada. 2014;8(3-4):E216-E20.

13. Oude Elferink P, Witjes JA. Blue-light cystoscopy in the evaluation of non-muscle-invasive bladder cancer. Therapeutic advances in urology. 2014;6(1):25-33.

14. Zhu CZ, Ting $\mathrm{HN}, \mathrm{Ng} \mathrm{KH}$, et al. A review on the accuracy of bladder cancer detection methods. Journal of Cancer. 2019;10(17):4038-4044.
15. Kranzbühler B, Bieri U, Poyet C, et al. MP08-06 efficiency of bladder wash cytology during surveillance of non-muscle invasive bladder cancer: Analysis in a contemporary patient cohort. Journal of Urology. 2018;199(4S):e98.

16. Smith ZL, Guzzo TJ. Urinary markers for bladder cancer. F1000prime reports. 2013;5:21.

17. Raitanen MP. The role of BTA stat Test in follow-up of patients with bladder cancer: results from Finn Bladder studies. World J Urol. 2008;26(1):45-50.

18. Chou R, Gore JL, Buckley D, et al. Urinary Biomarkers for Diagnosis of Bladder Cancer: A Systematic Review and Meta-analysis. Ann Intern Med. 2015;163(12):922-931.

19. Tabayoyong W, Kamat AM. Current Use and Promise of Urinary Markers for Urothelial Cancer. Current urology reports. 2018;19(12):96.

20. Yafi FA, Brimo F, Steinberg J, et al. Prospective analysis of sensitivity and specificity of urinary cytology and other urinary biomarkers for bladder cancer. Urologic Oncology: Seminars and Original Investigations. 2015;33(2):66.e25-66.e31.

21. Oeyen E, Hoekx L, De Wachter S. Bladder Cancer Diagnosis and Follow-Up: The Current Status and Possible Role of Extracellular Vesicles. Int J Mol Sci. 2019;20(4):pii: E821.

22. Soria F, Droller MJ, Lotan Y, et al. An up-to-date catalog of available urinary biomarkers for the surveillance of non-muscle invasive bladder cancer. World journal of urology. 2018;36(12):1981-1995.

23. Snowdon J, Boag S, Feilotter $\mathrm{H}$, et al. A pilot study of urinary microRNA as a biomarker for urothelial cancer. Can Urol Assoc J. 2013;7(1-2):2832.

24. Hosseini J, Golshan AR, Mazloomfard MM, et al. Detection of recurrent bladder cancer: NMP22 test or urine cytology? Urology journal. 2012;9(1):367-372.

25. Bochenek K, Aebisher D, Miedzybrodzka A, et al. Methods for bladder cancer diagnosis - The role of autofluorescence and photodynamic diagnosis. Photodiagnosis and photodynamic therapy. 2019;27:141148

26. Osaghae SO, Turner DTL. Photodynamic diagnosis of bladder cancer: Initial experience of a single UK center. African Journal of Urology. 2014;20(3):123-129.

27. Martoccia C, Zellweger M, Lovisa B, et al. Optical spectroscopy of the bladder washout fluid to optimize fluorescence cystoscopy with Hexvix. Journal of Biomedical Optics. 2014;19(9):1-6.

28. Ray ER, Chatterton K, Khan MS, et al. Hexylaminolaevulinate fluorescence cystoscopy in patients previously treated with intravesical bacille Calmette-Guerin. BJU Int. 2010;105(6):789-794.

29. Kriegmair M, Zaak D, Rothenberger KH, et al. Transurethral resection for bladder cancer using 5-aminolevulinic acid induced fluorescence endoscopy versus white light endoscopy. J Urol. 2002;168(2):475-478.

30. Aboumarzouk $\mathrm{O}$, Valentine $\mathrm{R}$, Buist $\mathrm{R}$, et al. Laser-induced autofluorescence spectroscopy: Can it be of importance in detection of bladder lesions? Photodiagnosis and photodynamic therapy. 2015;12(1):76-83. 